\title{
Massive Autaptic Self-Innervation of GABAergic Neurons in Cat Visual Cortex
}

\author{
Gábor Tamás, ${ }^{1,2}$ Eberhard H. Buhl, ${ }^{1}$ and Peter Somogyi ${ }^{1}$ \\ ${ }^{1}$ Medical Research Council, Anatomical Neuropharmacology Unit, Department of Pharmacology, University of Oxford, \\ Oxford OX1 3TH, United Kingdom, and 2Department of Comparative Physiology, József Attila University, Szeged, \\ Hungary H-6726
}

\begin{abstract}
Autapses are transmitter release sites made by the axon of a neuron on its own dendrites. We determined the numbers and precise subcellular position of autapses on different spiny and smooth dendritic cell types using intracellular biocytin filling in slices of adult neocortex.

Potential self-innervation was light microscopically assessed on 10 pyramidal cells, 7 spiny stellate cells, and 41 smooth dendritic neurons from cortical layers II-V. Putative autapses occurred on each smooth dendritic neuron and on seven pyramids, but not on spiny stellate cells. However, electron microscopic examination of all light microscopically predicted sites on pyramids $(n=28)$ showed only one case of self-innervation with two autapses on dendritic spines. Interneurons were classified by postsynaptic target distribution (Tamás et al., 1997) and all putative autapses of seven basket, three dendrite-targeting, and three double bouquet
\end{abstract}

cells were scrutinized. All basket and dendrite-targeting cells established self-innervation, the number of autapses being $12 \pm 7$ and $22 \pm 12$ (mean $\pm S D$ ), respectively; only one of the double bouquet cells formed autapses $(n=3)$. Basket cell autapses $(n=$ 74) were closer to the soma $(12.2 \pm 22.3 \mu \mathrm{m})$ than autapses established by dendrite-targeting cells $(51.8 \pm 49.9 \mu \mathrm{m} ; n=66)$.

The degree of self-innervation is cell type-specific. Unlike on spiny cells, autapses are abundant on GABAergic basket and dendrite-targeting interneurons, with subcellular location similar to that of synapses formed by the parent cell on other neurons. The extensive self-innervation may modulate integrative properties and/or the firing rhythm of the neuron in a manner temporally correlated with its own activity.

Key words: autapse; interneuron; neocortex; inhibition; pyramidal cell; feedback
Van der Loos and Glaser (1972) proposed the word "autapse" to describe a transmitter release site made by the axon of a neuron and its own somatodendritic domain. In addition to their original Golgi study in rabbit neocortex predicting the existence of autapses, possible autaptic contacts have been described in dog (Shkol'nik-Yarros, 1971) and rat (Peters and Proskauer, 1980; Preston et al., 1980) cerebral cortex, monkey neostriatum (DiFiglia et al., 1976), and cat spinal cord (Scheibel and Scheibel, 1971). Using intracellular markers, several groups also detected apparent self-innervating connections from various brain regions, such as substantia nigra (Karabelas and Purpura, 1980) and striatum (Park et al., 1980; Preston et al., 1980), but the above studies were based on light microscopic observations. Peters and Proskauer (1980) verified an autapse on a multipolar stellate cell by electron microscopy (EM), and more recently autapses were found on layer $\mathrm{V}$ pyramids in developing neocortex (Lübke et al., 1996), on a fast-spiking neocortical interneuron (Thomson et al., 1996), and on a hippocampal GABAergic basket cell (Cobb et al., 1997). Although autapses formed in cell cultures have been used extensively to study the physiology of synaptic mecha-

\footnotetext{
Received April 2, 1997; revised June 3, 1997; accepted June 6, 1997.

This project was supported by the Wellcome Trust and by European Community Grant BIO4-CT96-0585. G.T. was supported by the Oxford-Szeged Scholarship and the Blaschko Visiting Research Scholarship. E.H.B. holds a Medical Research Fellowship from Corpus Christi College (Oxford, UK). We thank Mr. J. D. B. Roberts and Mr. F. Kennedy for technical and photographic assistance.

Correspondence should be addressed to Gábor Tamás, Medical Research Council Anatomical Neuropharmacology Unit, University of Oxford, Mansfield Road, Oxford OX1 3TH, UK.

Copyright (C) 1997 Society for Neuroscience $\quad 0270-6474 / 97 / 176352-13 \$ 05.00 / 0$
}

nisms (Segal, 1991; Pan et al., 1993; Shi and Rayport, 1994), few proposals have been made for the functional significance of inhibitory autaptic innervation in vivo (neostriatum, Park et al., 1980; Aplysia buccal ganglia, White and Gardner, 1981).

Neocortical GABAergic cells generally have smooth dendrites and receive input from both pyramidal and nonpyramidal cells as well as from subcortical afferents (Kisvárday, 1992). In a series of experiments (Buhl et al., 1997; Tamás et al., 1997), we labeled synaptically coupled pairs of neurons from slices of the cat visual cortex. During the anatomical analysis of such biocytin-filled cell pairs, we found that a significant portion of the connections, as detected by light and electron microscopy, was formed between axons and the soma and dendrites of the same cell. We confirmed the results with preparations containing only one filled neuron. Using intracellular biocytin labeling and correlated light microscopy (LM) and EM, we determined the exact number of autapses on several neocortical cell types. The filling of synaptically coupled cell pairs also allowed the comparison of the number and position of synaptic junctions on a given postsynaptic cell with that of autapses established by the same presynaptic neuron.

Preliminary results have been published in abstract form (Tamás et al., 1995).

\section{MATERIALS AND METHODS}

Slice preparation. Adult female cats weighing 2.6-3.2 kg were deeply anesthetized with intramuscular injection of ketamine $(30 \mathrm{mg} / \mathrm{kg})$ and xylazine $(1 \mathrm{mg} / \mathrm{kg})$. When pain reflexes had ceased, a craniotomy was performed on each animal to expose the visual cortex. Subsequently, the animals were perfused with $\sim 1500 \mathrm{ml}$ of chilled and oxygenated artificial CSF (ACSF). After the removal of the dura, blocks of brain tissue including the dorsal and medial aspect of the lateral gyrus and containing areas 17 and 18 of the visual cortex were removed from both hemi- 
spheres, and the biopsies were then immersed in chilled ACSF. No attempt was made to locate the retinotopic position of labeled cells. Using a Vibroslice (Campden Instruments, Loughborough, UK), 400$\mu \mathrm{m}$-thick frontal slices were cut from the dissected visual areas and transferred to a recording chamber where they were maintained at $34-35^{\circ} \mathrm{C}$ on nylon mesh at the interface between oxygenated ACSF and a humidified atmosphere saturated with a mixture of $95 \% \mathrm{O}_{2}$ and $5 \%$ $\mathrm{CO}_{2}$. The ACSF that was used for electrophysiological recordings was composed of (in mM) $126 \mathrm{NaCl}, 3.0 \mathrm{KCl}, 1.25 \mathrm{NaH}_{2} \mathrm{PO}_{4}, 24 \mathrm{NaHCO}_{3}$, $2.0 \mathrm{MgSO}_{4}, 2.0 \mathrm{CaCl}_{2}$, and 10 glucose. During perfusion, cutting, and preincubation, all $\mathrm{NaCl}$ was replaced with equiosmolar sucrose $(252 \mathrm{~mm})$ to prevent passive chloride entry, which has been suggested to be responsible for neurotoxicity during slice preparation (Aghajanian and Rasmussen, 1989). The slices remained in the sucrose solution for $30 \mathrm{~min}$ before the perfusion medium was changed to normal ACSF.

Intracellular recordings. Recording electrodes were pulled from standard wall borosilicate tubing, filled with $2 \%$ biocytin in $1.5 \mathrm{M} \mathrm{KCH}_{3} \mathrm{SO}_{4}$, and beveled to a DC resistance of $80-150 \mathrm{M} \Omega$. Putative GABAergic neurons were identified by their physiological characteristics, for example, short-duration action potentials followed by large amplitude, fast afterhyperpolarizing potentials (fAHPs) (McCormick et al., 1985). Once a stable recording had been obtained, a search was made for cells displaying the electrophysiological properties of pyramidal and spiny stellate neurons. Capacitive coupling was eliminated on-line using a modified Axoprobe amplifier (Axon Instruments, Foster City, CA). Synaptic coupling was tested using on-line spike-triggered averaging while eliciting firing in the interneuron with either depolarizing current pulses or constant DC current injections. Recordings were obtained with an Axoprobe amplifier, which was operated in bridge mode. Experimental data were acquired using a PCM instrumentation recorder and stored on videotapes. Data analysis was then continued off-line by redigitizing the data at $5-20 \mathrm{kHz}$ using commercially available 12 bit analog-to-digital boards (Computerscope, RC Electronics, Santa Barbara, CA; and Labmaster, National Instruments, Newbury, UK) in conjunction with the Axograph (Axon Instruments), RC Electronics Computerscope, and Whole Cell Program (courtesy of Dr. J. Dempster, University of Strathclyde, Glasgow, UK) software packages.

Histological processing and anatomical evaluation. In most of the cases, depolarizing current pulses (0.1-0.5 nA) resulted in an adequate diffusion of biocytin, filling the recorded neurons. To avoid deformations, slices were sandwiched between two Millipore (Bedford, MA) filters and fixed in $2.5 \%$ paraformaldehyde, $1.25 \%$ (both w/v) glutaraldehyde, and $15 \%(\mathrm{v} / \mathrm{v})$ saturated picric acid in $0.1 \mathrm{M}$ phosphate buffer, $\mathrm{pH}$ 7.4, for 12-24 hr. The tissue processing was based on previously described procedures (Han et al., 1993). Briefly, after gelatin embedding, the slices were resectioned at a $60 \mu \mathrm{m}$ thickness, and the biocytin-filled cells were visualized by the avidin-biotinylated horseradish peroxidase method with diaminobenzidine as a chromogen. Sections were postfixed with $1 \%$ $\mathrm{OsO}_{4}$ and block-stained in $1 \%$ uranyl acetate.

After flat embedding into resin (Durcupan, Fluka, Buchs, Switzerland), recovered cells were reconstructed at $1250 \times$ magnification from the entire slice using the $60-\mu \mathrm{m}$-thick serial sections with the aid of a drawing tube attached to a light microscope. In general, axonal filling was of high quality, and a significant portion of the axon collateralization was contained within the slice (i.e., there were no signs of partially filled axonal branches, because all collaterals ended in fine terminal segments with varicosities or were cut at the surface of the slice). With the exception of one basket cell, the location of the postsynaptic cells in the central portion of the slice enabled us to perform full, or nearly full, reconstructions of the dendritic arbors. The entire somatodendritic surface of both presynaptic and postsynaptic cells was tested for close appositions with filled axons, each of which was traced back to the soma. Light micrographs at different focal depths were taken from all such close appositions and from characteristic axonal and dendritic patterns.

After light microscopy, axon-rich areas including all layers covered by the axonal field were reembedded for ultrathin sectioning. Serial sections ( $\sim 80 \mathrm{~nm}$ thick) were cut and mounted on single-slot Butvar- or Formvarcoated copper grids, contrasted with lead citrate, and used to test synaptic output connections of the identified neurons by EM. After determination of the distribution of unlabeled postsynaptic target elements, such as soma, dendritic shafts, or spines (for details, see Tamás et al., 1997), all light microscopically detected sites of close appositions between filled axons and labeled somata, dendrites, and spines were tested in serial electron microscopic sections. Although in some cases the identification of the postsynaptic membrane specialization was not fea- sible, owing to the electron opaque reaction end product, we identified synaptic and autaptic junctions based on the following criteria: (1) vesicle accumulation in the presynaptic axonal varicosity, and (2) rigid membrane apposition between the presynaptic and postsynaptic element with a characteristic widening of the extracellular space. With one exception (see Results), the synaptic or autaptic cleft could be recognized by tilting the sections with the goniometer of the electron microscope. Membrane apposition alone, even at the electron microscopic level, did not necessarily reflect the presence of a synaptic junction (see Fig. 2C). Moreover, to avoid underestimation of the number of somatic synapses or autapses, all filled somata were completely sectioned serially for electron microscopic analysis to trace the axonal branches, which may have been obscured by the opaque cell bodies. The dendritic distance of autapses from the soma was measured on two-dimensionally projected reconstructions of the cells, thus underestimating the real three-dimensional distances along the dendrites.

Statistical methods. Fisher's exact test and Pearson's $\chi^{2}$ test for heterogeneity were applied to determine whether the cell groups defined were homogeneous and/or overlapping (S-Plus, Statistical Sciences, Seattle, WA). The results obtained by the two tests were similar, although the $p$ values were consistently greater using Fisher's test; therefore, we give the $p$ values determined by that test. The nonparametric Mann-Whitney $U$ test was applied to compare the properties of the different cell types. Unless indicated otherwise, results are given as mean \pm SD.

\section{RESULTS}

Putative self-innervation was light microscopically assessed on 58 biocytin-filled neocortical cells, including 41 aspiny interneurons from layers II-V and 17 spiny cells from layers II-IV of the visual cortex (Table 1). Apparent self-innervation could be observed on each smooth dendritic neuron and on the majority of pyramidal cells, but spiny stellate cells did not have axon terminals apposed to their own somatodendritic surface. Interneurons were classified by electron microscopic examination of their unlabeled postsynaptic targets. All putative autapses of 13 smooth dendritic neurons, selected to represent different degrees of overlap between the dendritic and axonal arborization from each class, and all the possible autaptic junctions on pyramidal cells were subject to serial sectioning and EM. Altogether 172 light microscopically observed close appositions were tested by EM, and 145 autapses could be verified. In eight cases we observed membrane appositions without autaptic junctions.

It is highly unlikely that autapses were formed during the slicing and incubation procedures, because the occurrence and frequency of self-innervation was cell type-specific and did not depend on the density of axonal arborizations. Furthermore, some of the self-innervating axonal branches appeared to be specifically aligned with the dendrites of the cell, forming multiple autaptic junctions through large boutons that did not make synapses with other cells (see Figs. 3, 4).

Light microscopic prediction of autapses proved unreliable; the degree of discrepancy between the LM estimates and electron microscopically verifiable autaptic junctions depended on the type of neuron. In the case of basket cells (BCs) and dendritetargeting cells (DTCs), having smooth dendrites and large axon terminals, $68 \%(n=28)$ and $87 \%(n=47)$ of the boutons that appeared to contact the dendrites were confirmed to make autaptic junctions (see Figs. 2, 4, 6). On somata, 93\% $(n=13, \mathrm{BCs})$ and $84 \%$ ( $n=5$, DTCs) of the predicted junctions could be verified (see Figs. 2 and 6). The analysis of serial sections of dendritic sites revealed six (17\%, BCs) and seven (12\%, DTCs) additional release sites by boutons forming multiple autaptic junctions (see Fig. 6C). Moreover, 27 and 7 additional autapses were found on the somata ( 68 and $58 \%$ of all identified somatic autapses on BCs and DTCs), which could not be predicted, because the dense peroxidase product obscured boutons on and 
Table 1. The extent of self-innervation in cortical neurons

\begin{tabular}{|c|c|c|c|c|c|}
\hline & \multicolumn{5}{|l|}{ Cell type } \\
\hline & Basket & Dendrite-targeting & Double bouquet & Pyramidal & Spiny stellate \\
\hline No. of tested cells & 7 & 3 & 3 & 10 & 7 \\
\hline No. of putative self-innervating cells (LM) & 7 & 3 & 3 & 7 & 0 \\
\hline No. of close process appositions (LM) & 57 & 61 & 26 & 28 & 0 \\
\hline No. of self-innervating cells (EM) & 7 & 3 & 1 & 1 & 0 \\
\hline No. of autaptic junctions (EM) & 74 & 66 & 3 & 2 & 0 \\
\hline No. of autapses per cell (mean $\pm \mathrm{SD})$ & $12 \pm 7$ & $22 \pm 12$ & $1 \pm 1.73$ & $0.2 \pm 0.6$ & 0 \\
\hline No. of autapses per cell (range) & $3-23$ & $9-32$ & $0-3$ & $0-2$ & 0 \\
\hline Distance from the soma $\left(2 \mathrm{D}^{a} ; \mu \mathrm{m} ;\right.$ mean $\left.\pm \mathrm{SD}\right)$ & $12.2 \pm 22.3$ & $51.8 \pm 49.9$ & $68.5 \pm 35.7$ & 80 & - \\
\hline
\end{tabular}

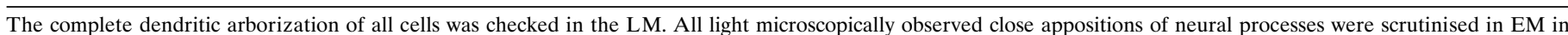

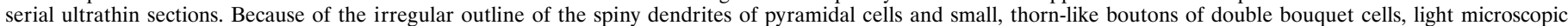

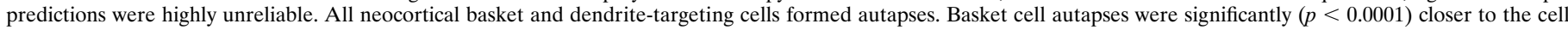

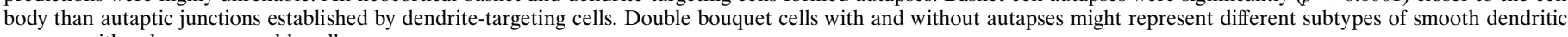
neurons with columnar axonal bundles.

${ }^{a}$ Measured as two-dimensional projected distance.

around the soma (see Fig. 6A). Although we predicted two and one autapses on axon initial segments of BCs and DTCs, none of these could be verified by EM (see Fig. $2 C$ ). The spiny dendrites of pyramidal cells have an irregular outline, and the dimensions of some of the spines are below the resolving power of the light microscope, hampering the prediction of contact with axons passing nearby. Consequently, $93 \%$ of predicted contacts between spiny dendrites and the axon of the neuron were not autapses when tested by EM (see Fig. 8). The characteristics of the axon also influenced the predictability of autapses, because the potential contacts between the small, thorn-like axonal boutons of double bouquet and pyramidal cells and their dendrites were difficult to evaluate in the light microscope. Thus, our large electron microscopically tested sample has led to a conclusion different from that of another correlated light and electron microscopic study based on eight electron microscopically tested autaptic sites (Lübke et al., 1996), which suggested a high reliability $(75 \%)$ of LM predictions.

\section{Autapses of basket cells}

BCs $(n=8)$ showed a postsynaptic target preference for somata $(49.0 \pm 11.8 \%)$ and dendritic shafts $(47.4 \pm 9.9 \%)$ and formed synapses occasionally with dendritic spines (3.6 \pm $4.5 \% ; n=135$ synapses; $n=26$ obtained in this study) (data on six cells from Tamás et al., 1997). LM observations indicated $8.1 \pm 3.7$ (range, 4-13) putative self-innervating contacts on the BCs. We determined the total number of autapses per cell on seven neurons from cortical layers II-IV showing similar dendritic trees but three types of axonal arborization and, therefore, different degrees of overlap between the axonal and dendritic tree. The dendrites of the remaining cell were not recovered, but we found four autapses on the soma, which was completely sectioned serially. Two neurons (1102943 and 0812942) distributed their axons mainly in layers II and III and established nine and three autapses; four cells (1102941, 2402942c1t2, 0812945b, and 2402942c1) sent collaterals to layers V and VI in addition to the extensive branching in layers II and III and formed 8 (Figs. $1 D, 2 B$ ), 8, 8, and 23 autaptic junctions. A large BC, with a patchy axon arbor in layers II-VI, established 15 autapses, although its axonal cloud was very sparse in the vicinity of the soma (Figs. $1 A-C, 2 A$ ). Overall, the number of electron microscopically verified autapses exceeded the LM predictions. The subcellular location of identified autapses was similar to the synaptic target distribution on other innervated neurons, because 40 autapses were found on somata $(5.7 \pm 4.9$ per cell; $47.3 \pm 33.1 \%)$, and 34 were found on proximal dendritic shafts $(4.9 \pm 4.1$ per cell; $52.7 \pm 33.1 \%$; Fig. $1 C, E)$. Moreover, although membrane apposition could be detected electron microscopically between a BC axon terminal and the initial segment of the axon of the cell, the bouton did not form an autaptic junction (Fig. 2C), indicating autaptic target preference toward the somatodendritic domain. This bouton formed a type II synapse on a neighboring cell body (Fig. 2C).

\section{Self-innervation by dendrite-targeting cells}

Based on their synaptic target preference ( $n=145$ synapses), six neurons were defined as DTCs (five cells from Tamás et al., 1997), innervating dendritic shafts predominantly $(81.3 \pm 11.0 \%)$ and occasionally dendritic spines $(14.9 \pm 10.8 \%)$ and somata $(3.8 \pm 4.6 \%)$. One cell was reconstructed for this study (Fig. 3$)$. Each DTC appeared to be strongly self-innervated on the basis of LM assessment (17.1 \pm 7.8 putative contacts; range, 6-26). To determine the total number of autapses on individual cells, a selection of three neurons had been made for EM from cortical layers II-IV, with different degrees of overlap between the dendritic and axonal trees. Regardless of the variability of the cell class, each dendrite-targeting interneuron massively innervated its somatodendritic domain. Cell 1003953 spanned all cortical layers with straight axonal branches giving rise to necklace-like

Figure 1. Self-innervation by BCs. $A-C$, Light microscopic reconstructions of a large BC in layer III of area 17 . The soma and dendrites are illustrated in red; axons are shown in black. Although the axonal cloud is relatively sparse in the neighborhood of the soma, the parent cell body is heavily innervated by high-order axonal branches. $B$, Route of the axon back to the parent soma. $C$, All electron microscopically verified autaptic junctions were located on, or very close to, the cell body. Three boutons established more than one autaptic junction, as indicated by grouped numbers. $D$, Autaptic junctions of a different type of BC in layer III. The dendritic arborization is shown in red; the axonal branches forming autapses are presented in black. The complete axonal arborization is illustrated by Buhl et al. (1997, their Fig. 3). E, Subcellular distribution of autaptic junctions. Both cells have higher autaptic target preference toward their soma than in the overall synaptic target distribution on other neurons. Asterisks indicate axonal branching points. 


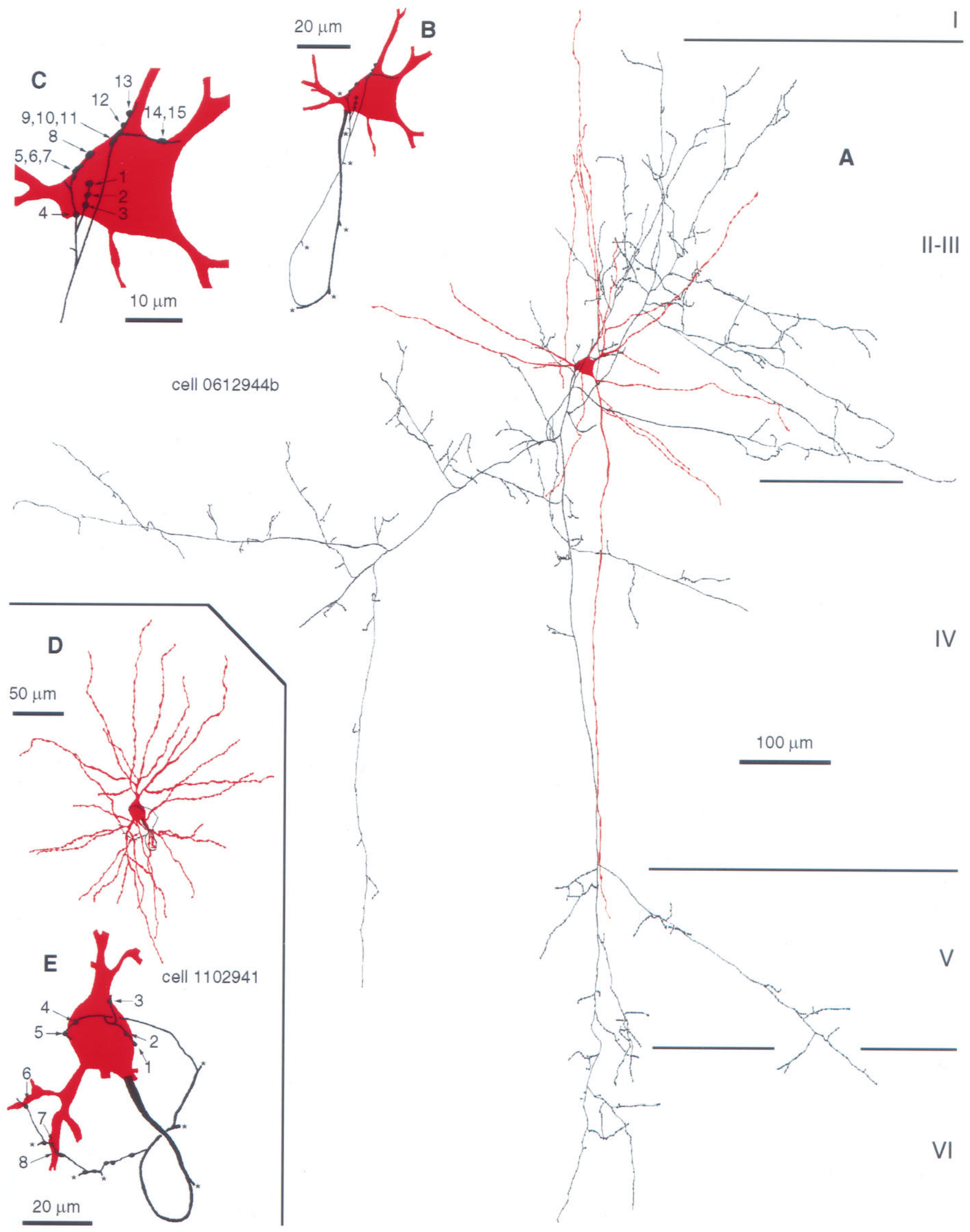




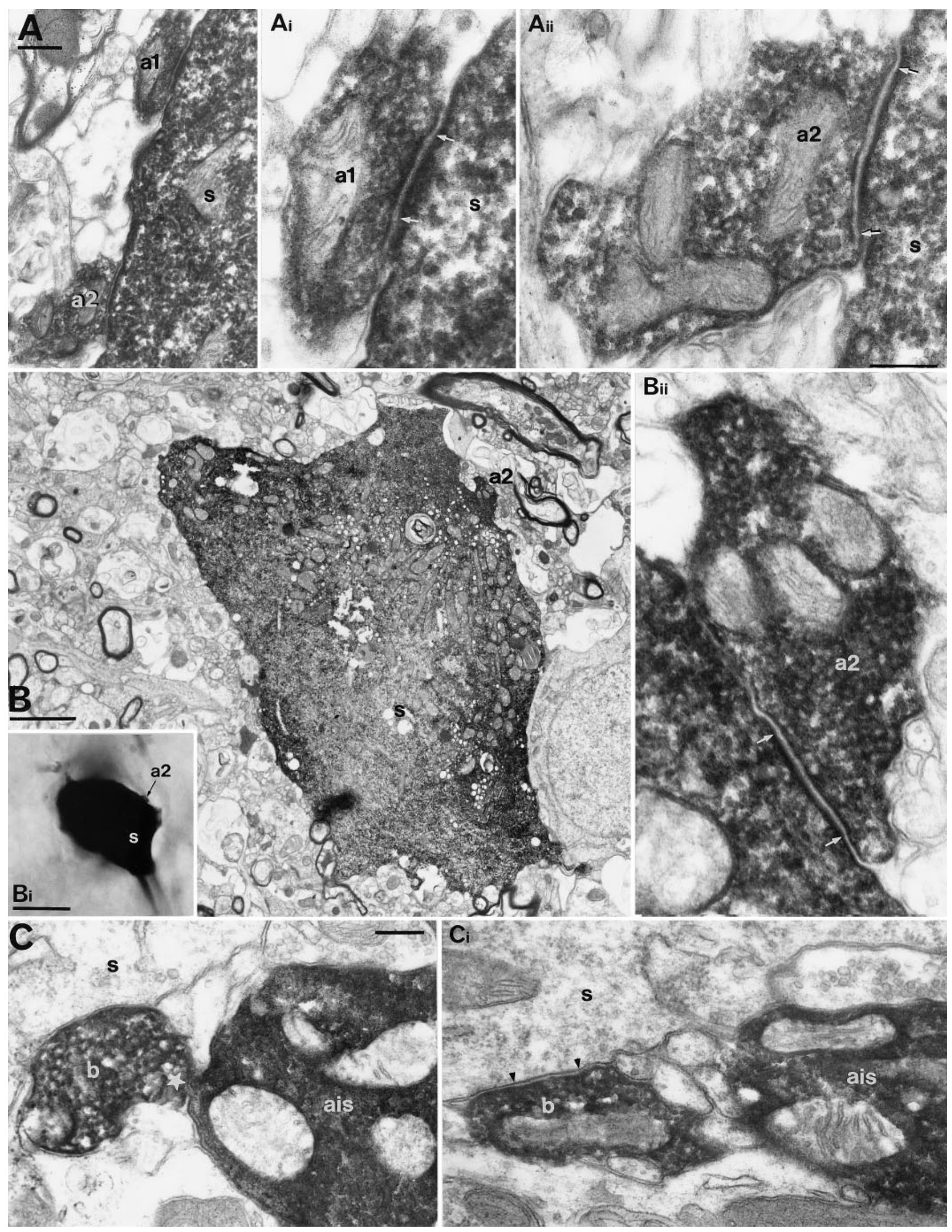




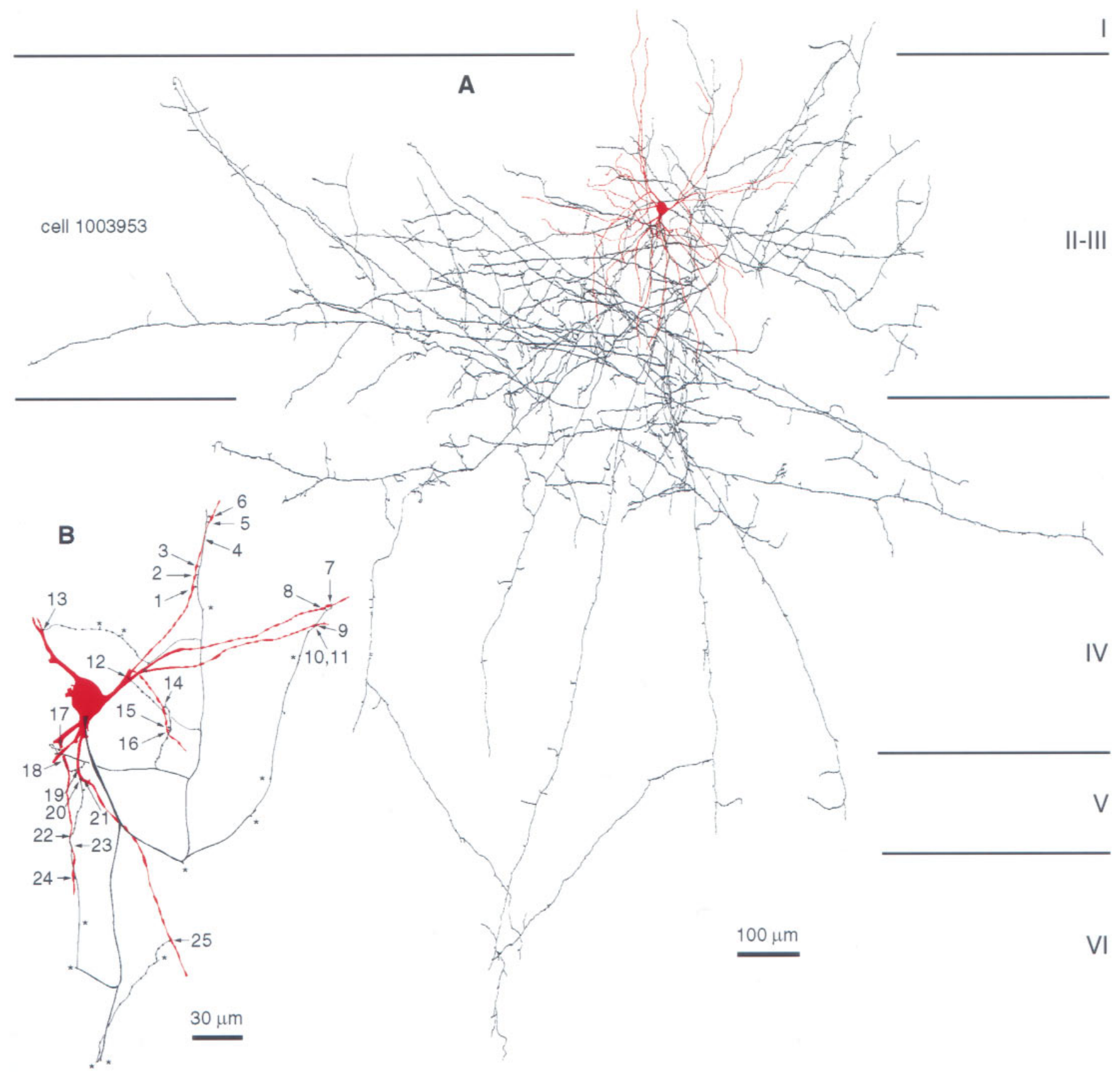

Figure 3. Exclusively dendritic self-innervation established by a DTC in area 18. A, Dendritic (red) and axonal (black) arborization of the DTC. B, Location of electron microscopically verified (see Fig. 4) autaptic innervation by boutons. All autapses were on dendrites at various distances from the soma. One bouton established two separate autaptic junctions $(10,11)$. The cell showed a synaptic target preference toward dendritic shafts $(72.7 \%)$ and also innervated spines $(27.3 \%)$ of other neurons. Asterisks indicate axonal branching points.

terminal segments mainly around the border between layers III and IV. The axonal arbor had a horizontal extent of $1460 \mu \mathrm{m}$ in the slice and established 25 autapses (Figs. 3, 4). A different, columnar axonal arborization was formed by cell 1506935 (for full reconstruction, see Tamás et al., 1997, their Fig. 6), which made 32 autapses (Figs. 5A, 6A,B). The third electron microscopically fully analyzed cell (1612941; for full reconstruction, see Buhl et al., 1997, their Fig. 1) had the bulk of its axonal terminals

$\leftarrow$

Figure 2. Electron microscopic demonstration of autaptic connections established by BCs. $A$, Two self-innervating boutons (a1, a2) on the soma (s) of the $\mathrm{BC}$ shown in Figure $1 A-C$. Ai, Aii, The autaptic junctions were identified by the rigid membrane apposition and the widening of extracellular space (between arrows) and the clustering of vesicles. Numbering is the same as in Figure $1 C$. B, Correlated LM (Bi) and EM of a self-innervating bouton (a2) targeting the soma $(s)$ of the parent BC 1102941. The autaptic junction is shown between arrows (Bii). C, A bouton $(b)$ of BC 0812942 forms a membrane apposition (star) with its own axon initial segment (ais) without autaptic specialization. In a serial section (Ci), the same bouton establishes a synaptic junction on an unlabeled soma (s). Scale bars: $A, A i, A i i, B i i, C, C i, 0.2 \mu \mathrm{m} ; A i$, Aii, Bii, same magnification; $C$, $C i$, same magnification; $B, 2 \mu \mathrm{m} ; B i, 10 \mu \mathrm{m}$. 


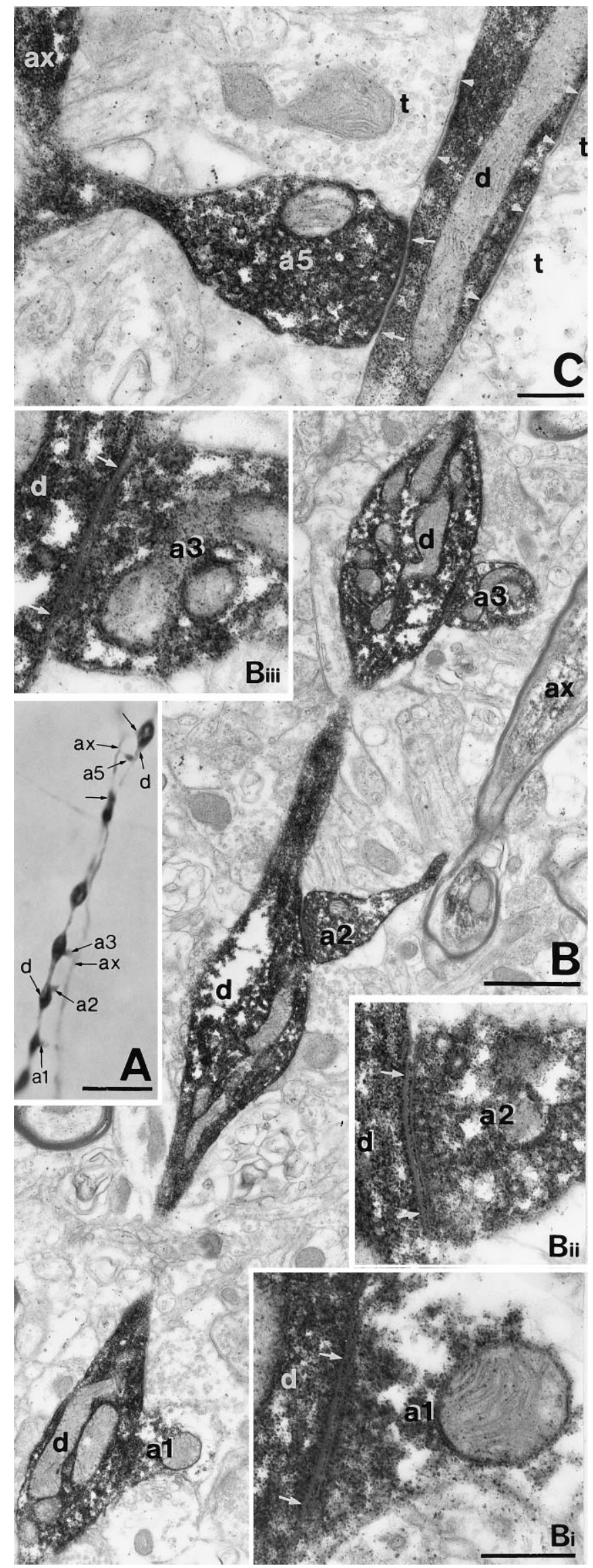

in layer IVa and established nine autaptic junctions (Figs. $5 B$, $6 C)$. Autapses on DTCs were located predominantly on dendrites $(n=54 ; 18.0 \pm 9.6$ per cell; $82.2 \pm 16.1 \%)$, and autaptic junctions on the parent somata were less frequent $(n=12 ; 4.0 \pm 5.3$ per cell; $17.8 \pm 16.1 \%$ ), a result similar to the overall synaptic target preference of the three neurons (Figs. 4, 6). Dendrite-targeting interneurons had a higher number of dendritic autapses than BCs ( $p<0.05$, Mann-Whitney $U$ test $)$, and, in addition, the distance of autaptic junctions from the soma, as measured in twodimensional projection, was significantly greater $(p<0.0001$; see Table 1).

\section{Autapses on double bouquet cells}

In the course of the experiments six double bouquet cells (DBCs) were recorded, but, despite extensive axonal filling, the dendrites were recovered from only three neurons. We then investigated the extent of self-innervation on the three fully recovered cells. Putative autapses were predicted by light microscopy on the dendrites of each DBC. On two cells, which had 8 and 12 suspected contact sites, none of the sites turned out to be autaptic junctions when tested by EM. On the third cell, three of six putative autapses could be verified by EM, and the autaptic junctions were on relatively distal dendrites (Table 1). It should be added that although the three cells were classified as DBCs, based on their characteristic axonal bundle traversing through all layers of the cortex (Somogyi and Cowey, 1981; Tamás et al., 1997), they showed a number of dissimilar characteristics. The dendrites of the two cells without autapses were thin and unbeaded and had branching points relatively distal (up to $100 \mu \mathrm{m}$ ) from the soma. In addition, the proximal region of these dendrites received characteristic invaginated asymmetrical synapses with a wide nonjunctional membrane apposition between the dendrite and the presynaptic terminals. The axons of these cells, and of all DBCs without recovered dendrites (Tamás et al., 1997), had small, thorn- or mushroom-like varicosities and thin interbouton segments and targeted preferentially dendritic spines (64.0 and $67.7 \%$, respectively) and less frequently dendritic shafts (36.0 and $32.3 \%$, respectively) (Tamás et al., 1997). In contrast, the dendrites of the additional DBC (not illustrated) analyzed in this study were strongly beaded, branching in the proximity of the soma and not receiving invaginated type I synapses. The axon of this autapse-forming cell was also different in that it had large, round terminals and thicker interbouton axon segments than the other DBCs. The random sample taken from unlabeled postsynaptic targets $(n=24)$ of this DBC showed a preference toward dendritic shafts $(62.5 \%)$ over dendritic spines $(37.5 \%)$. The postsynaptic target selectivity of the three DBCs tested here for autapses was heterogeneous (Fisher's exact test for heterogeneity, $p<0.05)$, but the two cells that formed no autapses were members of a homogeneous class of cells (Tamás et al., 1997).

\footnotetext{
Figure 4. Correlated LM and EM of autaptic junctions established by the DTC shown in Figure 3. A, A myelinated axonal branch (ax) gives rise to six en terminaux boutons (a1-a5 and unlabeled arrows) apposed to a distal dendrite $(d)$ of the parent cell. Unmarked arrows indicate autaptic boutons not shown by EM on this figure. $B$, Three of the autaptic boutons shown in $A$ are seen emerging from the myelinated axonal trunk $(a x)$ and target successive dendritic beads. Bi-Biii, The three autaptic junctions of $B$ are illustrated at higher magnification. $C$, A self-innervating bouton targeting the interbead segment of the parent dendrite is surrounded by unlabeled terminals $(t)$ establishing synapses (between arrowheads). The numbering is identical to Figure $3 B$. Autaptic clefts are indicated between arrows. Scale bars: $A, 10 \mu \mathrm{m} ; B, 1 \mu \mathrm{m} ; B i-B i i i$ (same magnification), $C, 0.3 \mu \mathrm{m}$.
} 


\section{Comparison of synaptic and autaptic innervations established by the same interneurons}

The number and location of autapses could be compared with those of identified synapses established by the same neuron. One of the DTCs (1506935) elicited fast IPSPs in a postsynaptic spiny stellate cell through three dendritic release sites, which were equidistant from the soma of the spiny stellate cell (see Tamás et al., 1997, their Fig. 6). The autaptic innervation was an order of magnitude higher in terms of the number of release sites $(n=32)$. Autapses were scattered over the surface of the parent cell, varying from relatively distal dendrites (Fig. $5 A$, a14) to the soma (Fig. 6A,B). It should be added, however, that as with DTC 1612941, all somatic autapses were formed by only one axon collateral, and the cell showed a moderate innervation of the somata of other neurons $(10.5 \%)$.

All autaptic and synaptic (Tamás et al., 1997) junctions were also tested electron microscopically from cell pair 1612941, consisting of a reciprocally connected layer III-IV border pyramidal cell and a layer IV DTC (Fig. 5B). Only the EPSPs, elicited by the pyramidal cell via five synapses, were measured in the DTC (for the recordings and complete axonal and dendritic arbors, see Buhl et al., 1997, their Fig. 1). The interneuron established multiple synaptic junctions $(n=17)$ on the pyramidal cell and also formed numerous autapses $(n=9)$; the subcellular location of synaptic and autaptic junctions was similar. The pyramid was innervated by two synaptic clusters on two basal dendrites (i6-i10 and i14-i17) and by a cluster on the soma near the origin of a dendrite (i1-i5). Autapses on the parent cell formed a cluster on the soma (a3-a4), two clusters (a5-a7 and a8-a9), and two solitary junctions (a1 and a2) on dendrites (Figs. 5B, 6C).

\section{Rare self-innervation of pyramidal cells}

We tested 10 cells from granular and supragranular layers, including one cell in layer IV, three in layers II and III, two small pyramids in layer II, and four layer III-IV border pyramidal cells. The local axonal arborizations varied from a narrow, columnartype distribution to a wider, fan-like arrangement, but in each case numerous axonal branches passed through the dendritic arbor. Although on seven cells close appositions could be observed in the light microscope between axons and the dendritic shafts $(n=17)$ and spines $(n=11)$ of the parent cell, only two autapses could be verified by EM on two neighboring spines of a layer III-IV border pyramidal cell (Figs. 7, 8). In addition, a membrane apposition between the same axon collateral forming the verified autapses and a basal dendritic shaft could not be evaluated reliably because of the almost parallel plane of the cutting and the potential autaptic cleft. On two other pyramidal cells, 2 nonautaptic membrane appositions were found on dendritic shafts.

\section{DISCUSSION}

The results demonstrate extensive self-innervation by at least two distinct classes of GABAergic cortical neurons, BCs and DTCs. We identified 32 autapses on a DTC, which represent the highest number of transmitter release sites so far on a single cortical cell originating from an individual axon of any origin (e.g., see Freund et al., 1985; Kisvárday et al., 1987; Deuchars et al., 1994; Thomson et al., 1996; Buhl et al., 1997). The formation of autapses is both cell type-specific and selective with regard to the placement of autapses on specific subcellular domains. A high level of self-innervation in the two cell classes is in contrast with its absence or rare occurrence in spiny stellate and pyramidal cells as well as in other types of GABAergic neurons. By testing 172 predicted sites we found that in many cases LM predictions for autapses are unreliable, and electron microscopic evaluation is essential to ascertain the presence of both direct membrane apposition and autaptic membrane specialization.

\section{Autapses in the CNS}

The existence of autapses has been reported primarily from cultures (Crain, 1971; Landis, 1976; Bekkers and Stevens, 1991; Segal, 1991), and their formation has been considered an artifact because of the lack of appropriate postsynaptic targets. Occasional autapses on neurons have been reported in anatomical studies from various brain regions (Held, 1897; Chan-Palay, 1971; Scheibel and Scheibel, 1971; Shkol'nik-Yarros, 1971; Van der Loos and Glaser, 1972; DiFiglia et al., 1976; Karabelas and Purpura, 1980; Peters and Proskauer, 1980; Preston et al., 1980; Kuffler et al., 1987; Shi and Rayport, 1994; Lübke et al., 1996), but in view of our difficulty predicting the existence of autapses on the basis of light microscopy, some of the previous predictions will require reexamination, including $\mathrm{EM}$ or physiology. To our knowledge, electron microscopic studies have so far verified one autapse on a smooth dendritic stellate cell (Peters and Proskauer, 1980), six autapses on layer V pyramidal cells (Lübke et al., 1996), one autaptic junction on a fast-spiking interneuron (Thomson et al., 1996), and five autapses on a hippocampal basket cell (Cobb et al., 1997).

\section{Self-innervation is cell type- and domain-specific}

It is possible that autapses may result from the chance meeting of the axon of the neuron with its somatodendritic surface. However, several observations suggest that this is not the case. First, the number of autapses per BC or DTC is at least three times higher than the number of potential synapses originating from a single interneuron on an individual postsynaptic cell, assuming that each cell in the axonal field receives an equal number of unitary synapses (Tamás et al., 1997). Second, the degree of autaptic innervation greatly varies between different types of cell, although there is a substantial overlap between the dendritic and axonal arborizations in all cell types tested. Although within the dendritic tree the density of GABAergic boutons originating from a single cell is higher than that of pyramidal and spiny stellate cells, this alone does not explain the high incidence of autapses, because axo-axonic cells do not form autapses (Somogyi et al., 1982; Freund et al., 1983), and DBCs only exceptionally seem to establish self-innervation despite the high density of their axons. Third, the subcellular domain-specific innervation of a cell seems to be preserved in the autaptic innervation; "forbidden" cell regions are not targeted by autapses, even when membrane apposition provides an opportunity. Finally, cells with a sparse axon cloud in their somatodendritic domain establish massive autaptic innervation.

Autapses on BCs are also placed close to their soma in the hippocampus of the rat (Cobb et al., 1997), and a multipolar stellate cell in the rat cortex, resembling BCs identified in this study, formed an autapse close to the soma (Peters and Proskauer, 1980). The DTC autapses were more distal than BC autapses, similar to the more distal location of their efferent synapses. Pyramidal cells, when they form autapses, do so on dendritic shafts and spines (Lübke et al., 1996), where their efferent synapses are found on other cortical cells (Kisvárday et al., 1986; Deuchars et al., 1994). Target zone preference might also contribute to the lack of autaptic innervation by some aspiny DBCs, 
cell pair 1612941

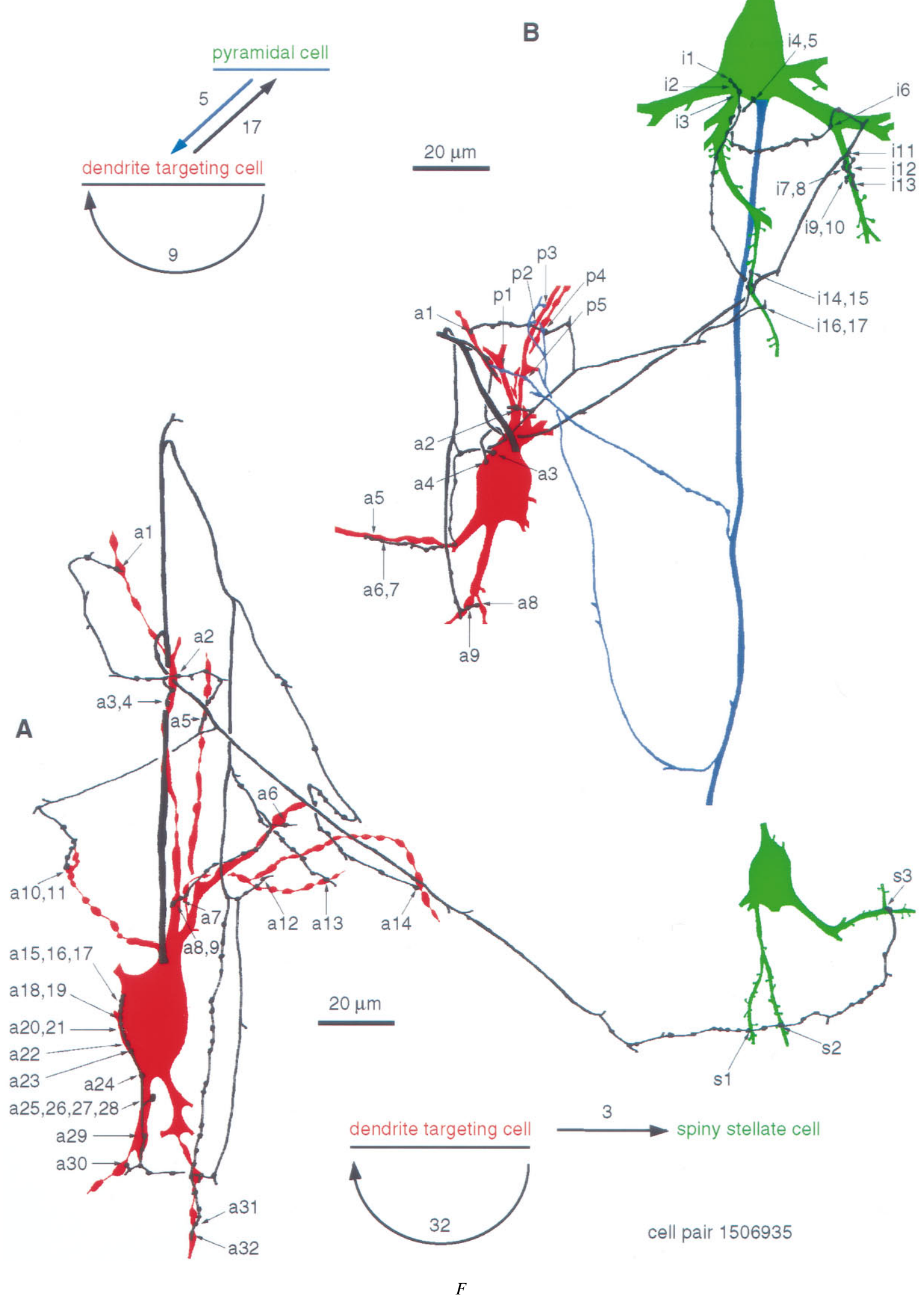



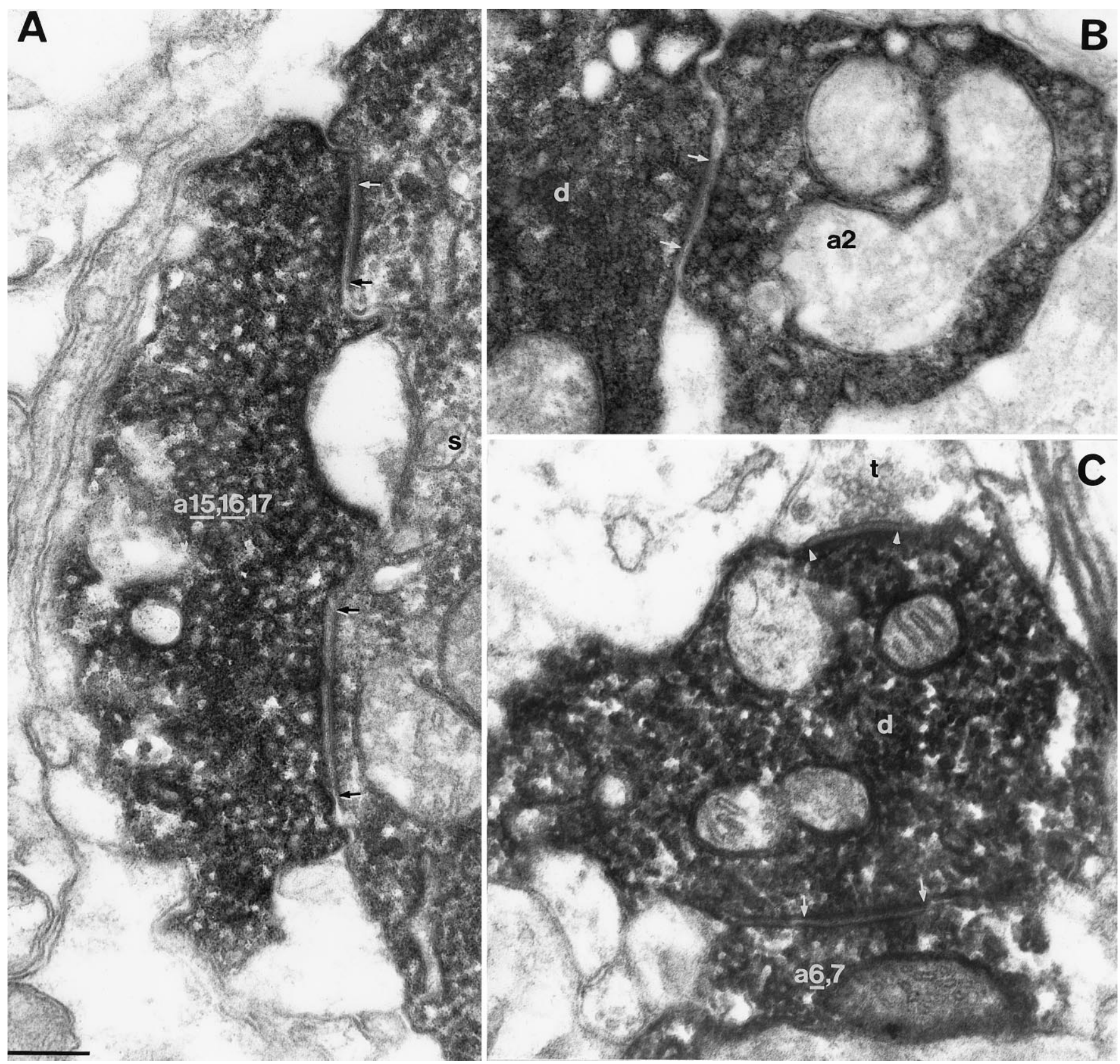

Figure 6. Examples of autaptic junctions established by the DTCs shown in Figure 5. $A$, $B$, Autapses on the DTC of Figure 5A. $A$, An autaptic terminal $(a \underline{15}, 16,17)$ targeting the soma $(s)$ established three separate autaptic junctions, two of which (underlined numbers) are illustrated here. $B, A$ bouton $(a 2)$ of the DTC forms an autapse on the dendrite of the cell. $C$, One (underlined number) of the two autapses established by a terminal $(a 6,7)$ of the DTC shown in Figure $5 B$. A synapse (between arrowheads) formed by an unlabeled bouton $(t)$ and the dendrite $(d)$ of the DTC are indicated. Numbering is identical to Figure 5. Autaptic clefts are indicated between arrows. Scale bar for all panels, $0.2 \mu \mathrm{m}$.

Figure 5. Comparison of synaptic and autaptic connections established by individual interneurons. $A$, Synaptic and autaptic connections of a layer IV DTC (soma and dendrites, red; axon, black) and a spiny stellate cell (soma and dendrites, green; axon not shown). The complete axonal and dendritic arborizations are shown by Tamás et al., (1997, their Fig. 6). The DTC innervated the spiny cell through three dendritic synapses (s1-s3) and established 32 autapses (a1-a32). Grouped numbers represent multiple autaptic junctions formed by the same bouton. Ten autapses were found on the soma, a higher proportion than the somatic targets $(10.7 \%)$ in the overall synaptic target distribution. $B$, Synaptic and autaptic relationships between a layer IV DTC (soma and dendrites, red; axon, black) and a layer III-IV border pyramidal cell (soma and dendrites, green; axon, blue). The complete axonal and dendritic arborizations are shown by Buhl et al., (1997, their Fig. 1). The cell pair was in reciprocal synaptic connection, but only the effect of the pyramidal cell could be recorded. The DTC established nine autaptic junctions (a1-a9), innervated the pyramidal cell via 17 synapses $(i 1-i 17)$, and received five synapses from the pyramid $(p 1-p 5)$. Note that the subcellular distribution of synaptic and autaptic junctions established by the interneuron was similar on both cells. 


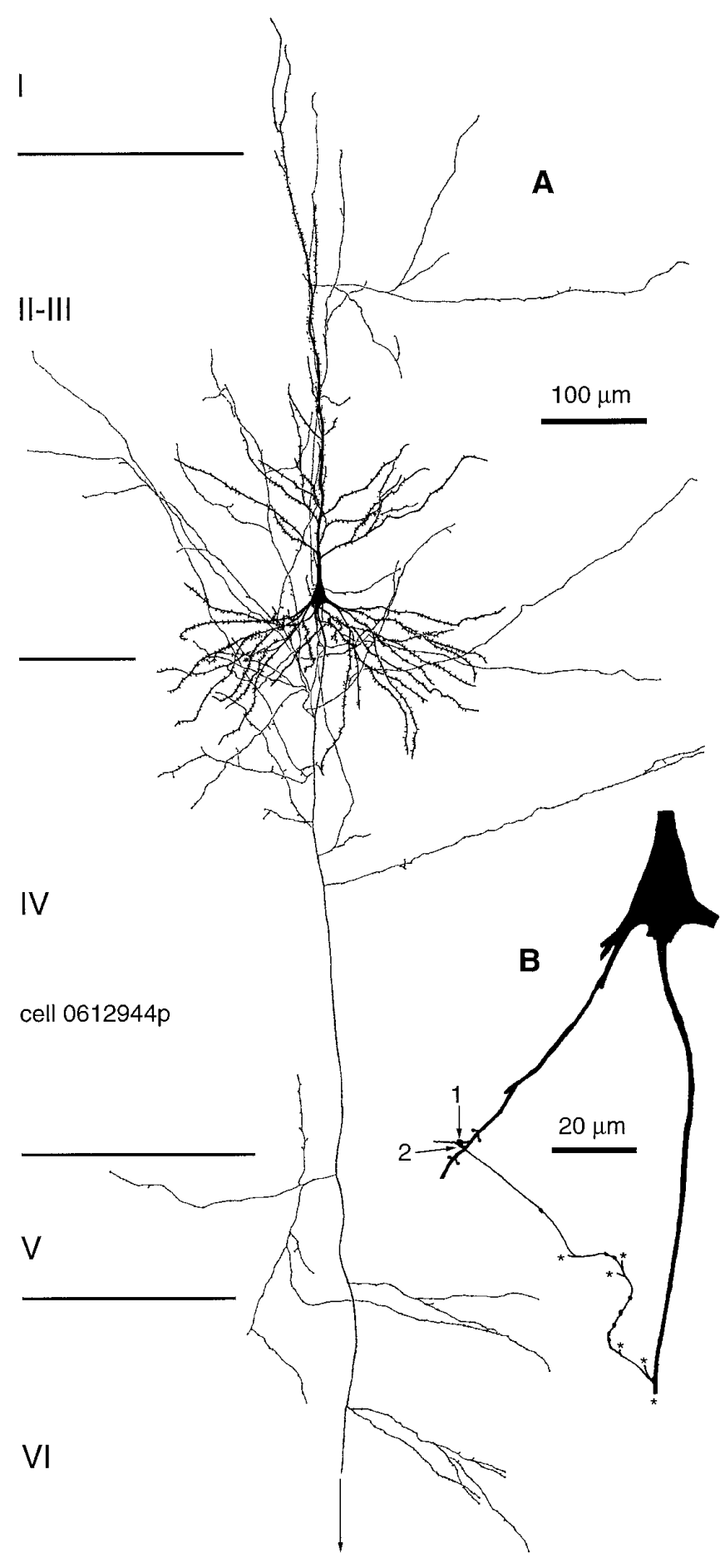

Figure 7. Reconstruction of the only self-innervating pyramidal cell of 10 tested. $A$, Dendritic and axonal arborization of the pyramidal cell from area 17. $B$, Location of the two electron microscopically verified autaptic junctions on the pyramidal cell. Autapses were formed by two neighboring boutons of a seventh order axon collateral on two adjacent dendritic spines on a fourth order dendrite. For correlated EM of the connection, see Figure 8. Asterisks indicate axonal branching points.

which terminate predominantly on dendritic spines and occasionally on shafts (Tamás et al., 1997). The preservation of subcellular specificity for the placement of transmitter release sites suggests a similar functional role for the autapses and synapses of the cell.

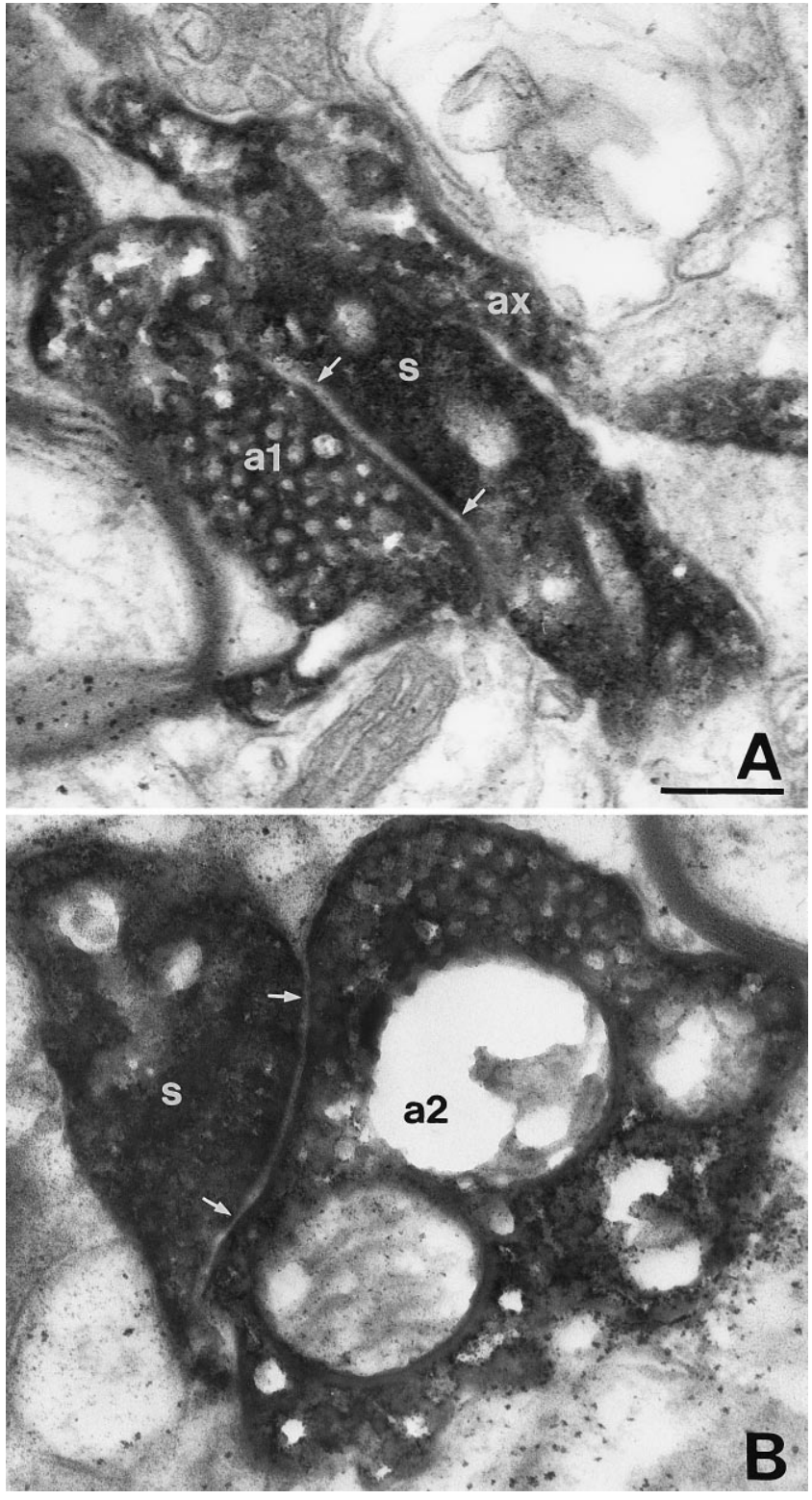

Figure 8. The autaptic junctions established by the pyramidal cell shown in Figure 7. $A, B$, The axon of the pyramidal cell $(a x)$ formed two closely located en terminaux autaptic boutons $(a 1, a 2)$, which innervated two neighboring dendritic spines $(s)$. Autaptic junctions are indicated between arrows. Scale bar for both panels, $0.2 \mu \mathrm{m}$.

Supragranular pyramidal cells in the adult cat cortex, with one exception, did not form autapses, in contrast to the reported presence of frequent autapses on layer $\mathrm{V}$ pyramids in the developing rat cortex (Lübke et al., 1996). Apart from the differences in species and the age of the animals, methodological factors may also explain the difference in conclusions, because in our study LM examination alone would have also led to a higher incidence of predicted autapses. However, pyramidal cells constitute many morphological and physiological subtypes (Chagnac-Amitai et al., 1990; Larkman and Mason, 1990), and further studies may reveal more extensive autaptic innervation in some of the types not examined here.

\section{Possible functional significance of autapses}

In cultured excitatory and inhibitory neurons (Bekkers and Stevens, 1991; Segal, 1991; Shi and Rayport, 1994) the effect of 
autapses is mediated by receptor mechanisms similar to those detected at efferent synapses of the same cell class. Indeed, Park et al., (1980) showed putative autaptic $\mathrm{GABA}_{\mathrm{A}}$ receptormediated shunting of evoked EPSPs after burst firing in GABAergic striatal medium spiny neurons. What functional differences require self-innervation for some GABAergic cells in the cortex, but not for others, remains unclear. It is conceivable, however, that differences in the firing pattern of interneurons having or lacking autapses may be shaped by the autaptic outward currents in a certain frequency range. Inhibitory autapses can prolong the initial interspike interval in Aplysia buccal ganglia (White and Gardner, 1981), maximize spike frequency, prevent burst firing, and contribute to spike afterhyperpolarization in cultured nucleus accumbens neurons (Shi and Rayport, 1994).

In the neocortex, neither excitatory nor inhibitory autaptic effects have been recorded, but the cell type specificity and the high number of autapses on some GABAergic cells predict effects that are functionally significant for the operation of these cells. It is not known how many synapses converge on a cortical GABAergic cell of any type, but in analogy with other cells a range of 1000-5000 seems realistic (Anderson et al., 1994). Taking a value of 3000 , the mean number of autapses being 13 , it is apparent that $0.4 \%$ of all transmitter release sites could originate from the axon of the neuron. If the proportion of GABAergic synapses in the input of BCs and DTCs is the same as in the overall population, then $17 \%$ of the synapses would be GABAergic (Beaulieu and Somogyi, 1990). On average, autapses would then provide $2.5 \%$ (range, 0.6-6\%) of the GABAergic input to BCs and DTCs. The amplitude of somatically recorded unitary IPSPs elicited by selfinnervating BCs and DTCs in postsynaptic pyramidal and spiny stellate cells was $1416 \pm 769 \mu \mathrm{V}$ (Tamás et al., 1997). Assuming that autapses have functional properties similar to synapses made by the same cell, a fast GABAergic effect could significantly influence local dendritic information processing and possibly also the firing properties of the cell.

Recent results indicate that IPSPs evoked in dendrites can modulate spike back-propagation and associated changes in intracellular calcium electrogenesis (Miles et al., 1996; Tsubokawa and Ross, 1996). It is not known whether action potentials are actively propagated in the dendrites of cortical GABAergic cells, but in GABAergic cells of the substantia nigra this might be the case (Häusser et al., 1995). Back-propagating action potentials and autaptic IPSPs would be tightly correlated and could arrive in synchrony at the dendrites. The relative timing would be influenced by the subcellular position of autapses and the length, myelination, and thickness of axonal branches connecting the autaptic terminals. Whether an interaction of possible backpropagating action potentials with tightly correlated IPSPs is necessary for the function of certain GABAergic neurons remains to be established, but it could be relevant in regulating coincidence between postsynaptic potentials and action potentials (Markram et al., 1997).

The generation of an IPSP of tens of milliseconds duration in the dendrites and soma of a neuron that fired an action potential as a result of synaptic drive may not initially have much effect, owing to the considerably larger conductance of the action potential and the fAHP, the latter being much shorter in BCs and DTCs than a conventional GABA $\mathrm{A}_{\mathrm{A}}$ receptor-mediated IPSP (Luhmann and Prince, 1991). Therefore, the late phase of the IPSP could potentially interact with other PSPs after the AHP. The effect of an increase in conductance during the autaptic IPSP may shorten EPSP duration, narrowing the window for summation and promoting coincidence detection (Softky, 1995; Buhl et al., 1997). Such a modulatory effect would be linked to the firing activity of the neuron, and the high number of autapses reported here could selectively influence the EPSPs that arrive temporally correlated with the firing of the cell.

\section{REFERENCES}

Aghajanian GK, Rasmussen K (1989) Intracellular studies in the facial nucleus illustrating a simple new method for obtaining viable motoneurons in adult rat brain slices. Synapse 3:331-338.

Anderson JC, Douglas RJ, Martin KAC, Nelson JC (1994) Map of the synapses formed with the dendrites of spiny stellate neurons of cat visual cortex. J Comp Neurol 341:25-38.

Beaulieu C, Somogyi P (1990) Targets and quantitative distribution of GABAergic synapses in the visual cortex of the cat. Eur $\mathbf{J}$ Neurosci 2:296-303.

Bekkers JM, Stevens CF (1991) Excitatory and inhibitory autaptic currents in isolated hippocampal neurons maintained in cell culture. Proc Natl Acad Sci USA 88:7834-7838.

Buhl EH, Tamás G, Szilágyi T, Stricker C, Paulsen O, Somogyi P (1997) Effect, number and location of synapses made by single pyramidal cells onto aspiny interneurones of cat visual cortex. J Physiol (Lond) 500:689-713.

Chagnac-Amitai Y, Luhmann HJ, Prince DA (1990) Burst generating and regular spiking layer 5 pyramidal neurons of rat neocortex have different morphological features. J Comp Neurol 296:598-613.

Chan-Palay V (1971) The recurrent collaterals of Purkinje cell axons: a correlated study of rat's cerebellar cortex with electron microscopy and the Golgi-method. Z Anat Entwicklungsgesch 134:210-234.

Cobb SR, Halasy K, Vida I, Nyíri G, Tamás G, Buhl EH, Somogyi P (1997) Synaptic effects of identified interneurons innervating both interneurons and pyramidal cells in the rat hippocampus. Neuroscience 79:629-648.

Crain SM (1971) Intracellular recordings suggesting synaptic functions in chick embryo spinal sensory ganglion cell isolated in vitro. Brain Res 26:188-191.

Deuchars J, West DC, Thomson AM (1994) Relationships between morphology and physiology of pyramid-pyramid single axon connections in rat neocortex in vitro. J Physiol (Lond) 478:423-435.

DiFiglia M, Pasik P, Pasik T (1976) A Golgi study of neuronal types in the neostriatum of monkeys. Brain Res 114:245-256.

Freund TF, Martin KAC, Smith AD, Somogyi P (1983) Glutamate decarboxylase-immunoreactive terminals of Golgi-impregnated axoaxonic cells and of presumed basket cells in synaptic contact with pyramidal neurons of the cat's visual cortex. J Comp Neurol 221: 263-278.

Freund TF, Martin KAC, Somogyi P, Whitteridge D (1985) Innervation of cat visual areas 17 and 18 by physiologically identified X-and Y-type thalamic afferents. II. Identification of postsynaptic targets by GABA immunocytochemistry and Golgi impregnation. J Comp Neurol 242:275-291.

Han Z-S, Buhl EH, Lörinczi Z, Somogyi P (1993) A high degree of spatial selectivity in the axonal and dendritic domains of physiologically identified local-circuit neurones in the dentate gyrus of the rat hippocampus. Eur J Neurosci 5:395-410.

Häusser M, Stuart G, Racca C, Sakmann B (1995) Axonal initiation and active dendritic propagation of action potentials in substantia nigra neurones. Neuron 15:637-647.

Held H (1897) Beiträge zur Struktur der Nervenzellen und ihrer Fortsätze. Arch Anat Physiol 2:204-294.

Karabelas AB, Purpura DP (1980) Evidence for autapses in the substantia nigra. Brain Res 200:467-473.

Kisvárday ZF (1992) GABAergic networks of basket cells in the visual cortex. Prog Brain Res 90:385-405.

Kisvárday ZF, Martin KAC, Freund TF, Maglóczky Z, Whitteridge D, Somogyi P (1986) Synaptic targets of HRP-filled layer III pyramidal cells in the cat striate cortex. Exp Brain Res 64:541-552.

Kisvárday ZF, Martin KAC, Friedlander MJ, Somogyi P (1987) Evidence for interlaminar inhibitory circuits in the striate cortex of the cat. J Comp Neurol 260:1-19.

Kuffler DP, Nicholls J, Drapeau P (1987) Transmitter localization and 
vesicle turnover at a serotoninergic synapse between identified leech neurons in culture. J Comp Neurol 256:516-526.

Landis SC (1976) Rat sympathetic neurons and cardiac myocytes developing in microcultures: correlation of the fine structure of endings with neurotransmitter function in single neurons. Proc Natl Acad Sci USA 73:4220-4224.

Larkman A, Mason A (1990) Correlations between morphology and electrophysiology of pyramidal neurons in slices of rat visual cortex. I. Establishment of cell classes. J Neurosci 10:1407-1414.

Lübke J, Markram H, Frotscher M, Sakmann B (1996) Frequency and dendritic distribution of autapses established by layer 5 pyramidal neurons in the developing rat neocortex: comparison with synaptic innervation of adjacent neurons of the same class. J Neurosci 16:3209-3218.

Luhmann HJ, Prince DA (1991) Postnatal maturation of the GABAergic system in rat neocortex. J Neurophysiol 65:247-263.

Markram H, Lübke J, Frotscher M, Sakmann B (1997) Regulation of synaptic efficacy by coincidence of postsynaptic APs and EPSPs. Science 275:213-215.

McCormick DA, Connors BW, Lighthall JW, Prince DA (1985) Comparative electrophysiology of pyramidal and sparsely spiny stellate neurons of the neocortex. J Neurophysiol 54:782-806.

Miles R, Tóth K, Gulyás AI, Hájos N, Freund TF (1996) Differences between somatic and dendritic inhibition in the hippocampus. Neuron 16:816-823.

Pan ZZ, Tong G, Jahr CE (1993) A false transmitter at excitatory synapses. Neuron 11:85-91.

Park MR, Lighthall JW, Kitai ST (1980) Recurrent inhibition in the rat neostriatum. Brain Res 194:359-369.

Peters A, Proskauer CC (1980) Synaptic relationships between a multipolar stellate cell and a pyramidal neuron in the rat visual cortex. A combined Golgi-electron microscope study. J Neurocytol 9:163-183.

Preston RJ, Bishop GA, Kitai ST (1980) Medium spiny neuron projection from the rat striatum: an intracellular horseradish peroxidase study. Brain Res 183:253-63.
Scheibel ME, Scheibel AB (1971) Inhibition and the Renshaw cell. A structural critique. Brain Behav Evol 4:53-93.

Segal MM (1991) Epileptiform activity in microcultures containing one excitatory hippocampal neuron. J Neurophysiol 65:761-770.

Shi WX, Rayport S (1994) GABA synapses formed in vitro by local axon collaterals of nucleus accumbens neurons. J Neurosci 14:4548-4560.

Shkol'nik-Yarros EG (1971) Neurons and interneuronal connections of the central visual system. New York: Plenum.

Softky WR (1995) Simple codes versus efficient codes. Curr Opin Neurobiol 5:239-247.

Somogyi P, Cowey A (1981) Combined Golgi and electron microscopic study on the synapses formed by double bouquet cells in the visual cortex of the cat and monkey. J Comp Neurol 195:547-566.

Somogyi P, Freund TF, Cowey A (1982) The axo-axonic interneuron in the cerebral cortex of the rat, cat and monkey. Neuroscience 7:2577-2607.

Tamás G, Buhl EH, Somogyi P (1995) High degree of self-innervation by GABAergic basket cells in the visual cortex of the cat as revealed in vitro. J Physiol (Lond) 487:63P.

Tamás G, Buhl EH, Somogyi P (1997) Fast IPSPs elicited via multiple synaptic release sites by distinct types of GABAergic neuron in the cat visual cortex. J Physiol (Lond) 500:715-738.

Thomson AM, West DC, Deuchars J (1996) Single axon IPSPs elicited in pyramidal cells by three classes of interneurones in slices of rat neocortex. J Physiol (Lond) 496:81-102.

Tsubokawa H, Ross WN (1996) IPSPs modulate spike backpropagation and associated $\left[\mathrm{Ca}^{2+}\right]_{\mathrm{i}}$ changes in the dendrites of hippocampal CA1 pyramidal neurons. J Neurophysiol 76:2896-2906.

Van der Loos H, Glaser EM (1972) Autapses in neocortex cerebri: synapses between a pyramidal cell's axon and its own dendrites. Brain Res 48:355-360.

White RL, Gardner D (1981) Self-inhibition alters firing patterns of neurons in Aplysia buccal ganglia. Brain Res 209:77-93. 